\title{
La tolérance néonatale aux allo-antigènes
}

Véronique Flamand

> Au début de la vie, notre système immunitaire possède des caractéristiques de tolérance face aux allo-antigènes. Durant cette période, notre immunité adaptative n'est pas au repos. Elle est au contraire polarisée de manière à favoriser l'activation de lymphocytes $T$ de type Th2 (T helper 2), au détriment de réponses cytotoxiques de type Thl ou Th17. Ceci pourrait expliquer la vulnérabilité des nouveau-nés face à l'agression par certains pathogènes, mais également leur sensibilité accrue au développement de pathologies allergiques ou leur faible réponse à certains vaccins. En fonction des facteurs environnementaux, cette tolérance néonatale sera régulée par d'autres acteurs immunitaires et aura ainsi des répercussions potentielles sur l'apparition ultérieure d'allergies ou de maladies auto-immunes. <

\section{Historique : la reconnaissance du soi et du non-soi}

La capacité de notre système immunitaire à reconnaître et à éliminer des corps étrangers, ou alloantigènes, s'élabore progressivement au cours de la vie intra- et extra-utérine. Globalement, les réponses immunitaires du fœtus et du nouveau-né ont été qualifiées d'immatures, tant sur des critères quantitatifs (nombre réduit de cellules immunitaires effectrices au sein des organes lymphoïdes périphériques) que qualitatifs (induction d'une immunité biaisée ou de tolérance, plutôt que de cytotoxicité). Comme nous le verrons, cet état peut rendre compte d'une plus grande susceptibilité aux infections et d'une qualité moindre de certaines réponses vaccinales.

En immunologie, la tolérance se définit comme une acceptation d'antigènes reconnus dans des circonstances particulières par les lymphocytes effec-

Vignette (Photo (c) Inserm - Behazine Combadière).

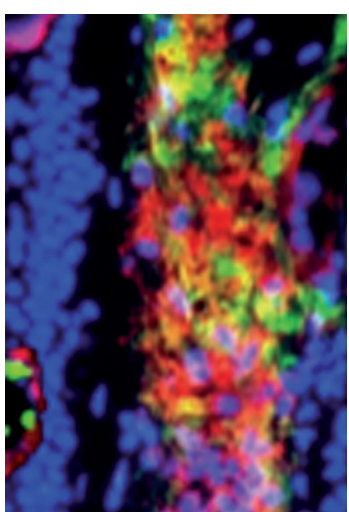

Institut d'immunologie médicale, Université libre de Bruxelles, 8 , rue Adrienne Bolland, 6041 Gosselies, Belgique. vflamand@ulb.ac.be

teurs du système immunitaire. En 1945, le Dr R.D. Owen découvrit fortuitement la tolérance néonatale grâce à une particularité anatomique naturelle de jumeaux bovins. Ce virologiste décrivit les conséquences immunologiques du développement d'embryons dizygotes ayant une circulation sanguine commune par anastomose de la vascularisation placentaire [1]. Les cellules échangées durant la transfusion croisée fœtale s'installèrent dans les tissus de l'autre jumeau et s'y maintinrent sans adaptation antigénique. Ce chimérisme hématopoïétique persista toute la vie de l'animal. L'argument majeur qu'une réelle reconnaissance antigénique avait eu lieu in utero se basa sur la constatation d'une absence définitive de réaction à une transfusion sanguine d'un veau à son jumeau, alors qu'une telle intervention sur des veaux nés d'une autre mère conduisait au rejet. Le phénomène « 0 wen » fut ensuite reproduit chez le poulet grâce à une méthode ingénieuse de parabiose synchoriale entre des embryons de poulet. En grandissant, chacun des poulets ainsi traités fut incapable, non seulement de synthétiser des anticorps contre les cellules de l'autre, mais également de rejeter une greffe de peau de l'autre individu [2].

Cette découverte clé alimenta la réflexion d'un virologiste australien, le Dr F.M. Burnet, pour qui les fonctions de notre immunité ne devaient pas se limiter à une défense contre une infection par des microorganismes pathogéniques. Il émit ainsi, en 1949, un premier postulat selon lequel le phénomène de tolérance et de non-antigénicité des composants du soi était la raison d'être de notre immunité, permettant la sauvegarde de notre intégrité et la survie des espèces animales. Notre système immunitaire serait doté d'une double reconnaissance lui permettant à la fois de déclencher une réponse de défense et de rejet contre toute entité étrangère (le non-soi), et de développer une auto-réactivité naturelle non destructrice contre nos 


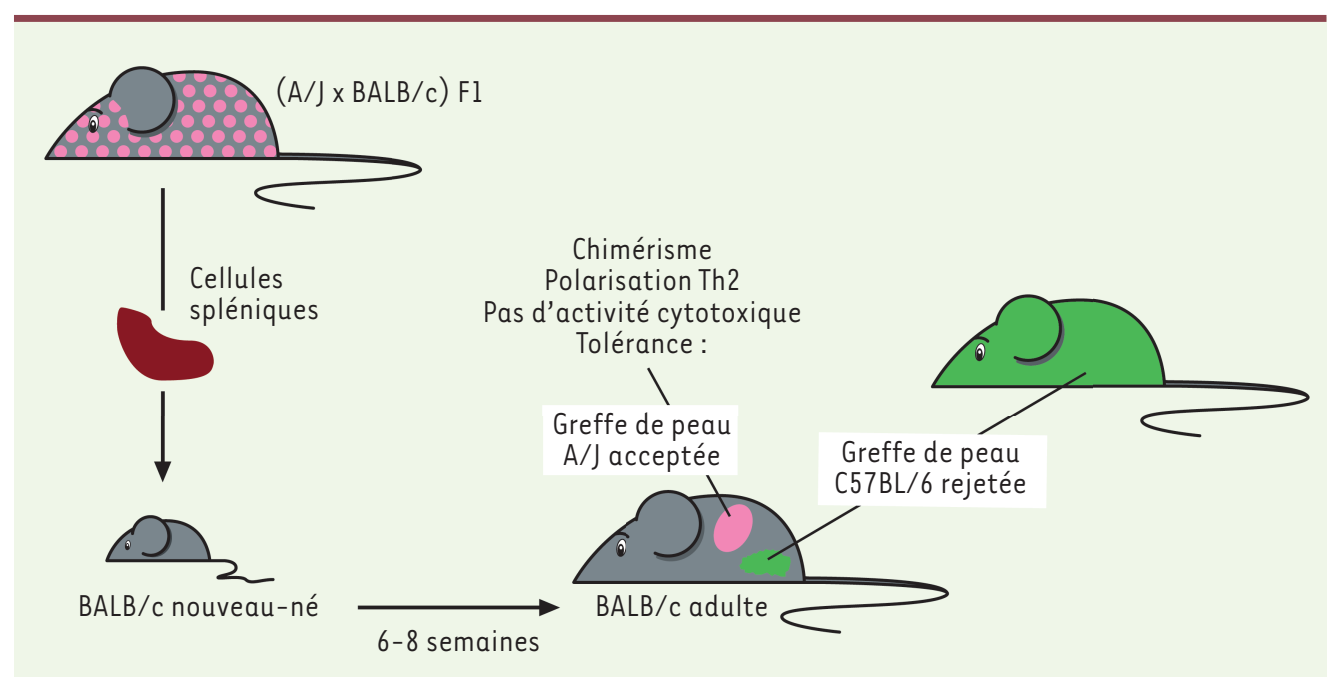

Figure 1. Le modèle expérimental de tolérance néonatale. Des souris de la souche $B A L B / c$ sont immunisées le jour de leur naissance avec des cellules spléniques de souris issues d'un croisement des souches $A / J$ et $B A L B / c$. Suite à cette stimulation allogénique, les lymphocytes $\mathrm{TCD}^{+}$du receveur nouveau-né se différencient en lymphocytes Th2 producteurs d'IL-4, d'IL-5 et d'IL-13. Un chimérisme lymphoïde s'établit et peut provoquer, à l'âge adulte, une pathologie de type Th2 caractérisée par une hyperplasie et une éosinophilie des organes lymphoïdes, ainsi qu'une hyperproduction d'lge. Les lymphocytes $T \operatorname{CD} 8^{+}$sont dépourvus de cytotoxicité envers les allo-antigènes rencontrés à la naissance. Enfin, une tolérance de transplantation de tissus exprimant les allo-antigènes $A / J$ s'observe, alors que ces animaux manipulés conservent la capacité de rejeter des tissus exprimant d'autres allo-antigènes (C57BL/6) [12].

propres composants (le soi). Des anomalies dans l'induction ou le maintien de la tolérance vis-à-vis du soi conduiraient à une réponse immune toxique appelée, par un des fondateurs de l'immunologie, le Dr P. Ehrlich, horror autotoxicus, contre nos antigènes autologues, conduisant aux maladies auto-immunes.

Dans ce contexte intellectuel initié par Owen et Burnet, les expériences réalisées par le biologiste britannique, P.B. Medawar, entre 1950 et 1953, allaient permettre de définir pleinement le phénomène de tolérance néonatale. P.B. Medawar démontra que, si des cellules vivantes (issues de la moelle osseuse ou de la rate) d'une souche de souris nommée CBA étaient injectées dans une souris adulte de souche $A$, les cellules CBA étaient détruites par un processus immunologique, et que la souris $A$ ayant subi cette transfusion détruisait d'autant plus efficacement toute greffe ultérieure ayant la même origine CBA. En revanche, si les cellules CBA étaient injectées in utero ou à un nouveau-né de la souche $A$, elles étaient acceptées. Qui plus est, en grandissant, la souris A ainsi traitée acceptait toute greffe ultérieure de la souche donneuse CBA comme si elle était sienne.

Billingham, Brent et Medawar proposèrent leur définition de l'acquisition active de la tolérance immunitaire comme étant une inhibition spécifique de la réponse immunitaire induite lorsqu'un individu est confronté durant sa vie fœtale à des cellules allogéniques [3]. Ils décrivirent ainsi une « période nulle » de transition entre la vie intrautérine et la vie extra-utérine, durant laquelle toute exposition à un stimulus antigénique conduisait à un état de faiblesse immunitaire spécifique ou de suppression de réactivité, et ce avant l'apparition d'une réelle faculté d'induction de réponse immunitaire.

Un point majeur à propos des conditions expérimentales d'induction de tolérance immunitaire néonatale était l'établissement d'un chimérisme cellulaire permanent. En effet, la source cel- lulaire allogénique, contenant des cellules progénitrices hématopoïétiques, devait rester présente même en quantité inférieure au seuil de détection, si un état de tolérance devait être maintenu. Cependant, les cellules lymphoïdes greffées issues de certaines lignées de souris possédaient toutes les qualités pour attaquer les tissus de l'hôte, pouvant conduire à une maladie chronique ou à la mort. Cette réactivité du greffon contre l'hôte put être évitée en injectant des cellules embryonnaires ou des cellules lymphoïdes issues de la première génération d'un croisement entre la souche CBA et $A$. Dans cette version du modèle qui fut utilisée pendant des décennies, le donneur et le receveur étaient tous deux immunoincompétents, le donneur pour la tolérance vis-à-vis du soi et le receveur pour l'immaturité de son système immunitaire (Figure 1).

Ainsi, Medawar fut reconnu pour ses travaux pionniers dans le domaine de l'induction de tolérance vis-à-vis des cellules allogéniques et Burnet pour sa définition de la reconnaissance immunitaire du soi. Tous deux furent corécipiendaires du prix Nobel de médecine en 1960. Leurs découvertes constituent la base de la compréhension des mécanismes immunitaires activés lors de transplantations d'organes.

\section{Les mécanismes de la tolérance néonatale}

Les mécanismes de la tolérance néonatale sont régis essentiellement par les lymphocytes $T$ qui 


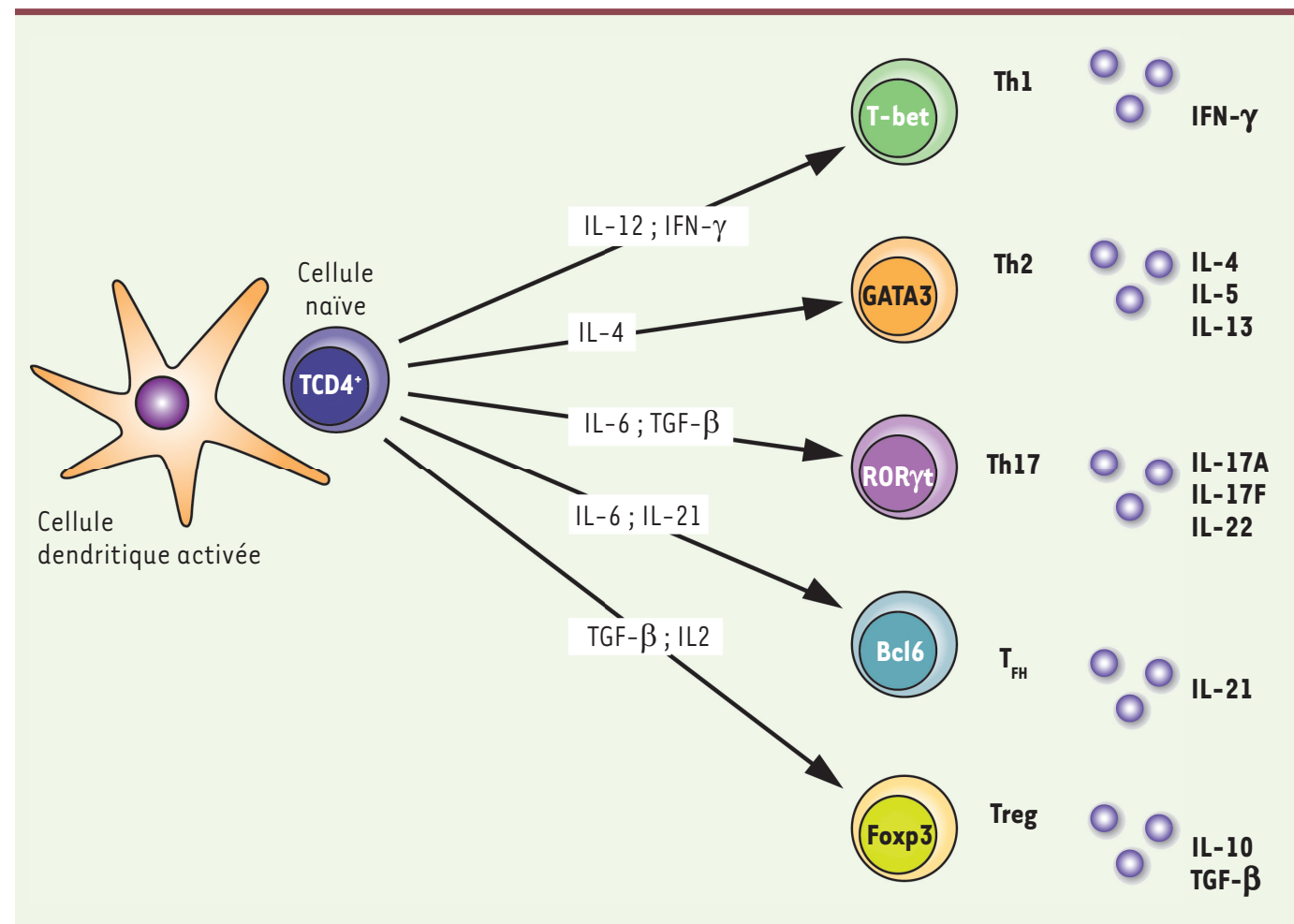

Figure 2. Les souspopulations de lymphocytes T CD4+. Suite à une rencontre antigénique, les lymphocytes $\mathrm{T} \mathrm{CD}^{+}$naïfs peuvent se différencier, soit en lymphocytes Thl en réponse à I'IL-12 et à I'IFN- $\gamma$ et produire de I'IFN- $\gamma$ grâce au facteur de transcription T-bet; soit en lymphocytes Th2 en réponse à I'IL-4 et produire de I'IL-4, de I'IL-5 et de I'IL-13 grâce au facteur de transcription Gata3; soit en lymphocytes Th17 en réponse à I'IL-6 et au TGF- $\beta$ et produire de I'IL-17A, IL-17F et de l'IL-22 grâce au fac-

teur de transcription RORyt (orphan nuclear receptor $\gamma t$ ) ; soit en lymphocytes $T_{F H}$ en réponse à l'IL-6 et à l'IL-2l et produire de l'IL-21 grâce au facteur de transcription Bcl6 (B-cell lymphoma 6); soit en lymphocytes Treg Foxp3+ en réponse au TGF- $\beta$ et à l'IL-2 et produire de l'IL-10 et du TGF- $\beta$. Les lymphocytes Thl permettent l'élimination de virus et de bactéries intracellulaires. Les lymphocytes Th2 participent à l'élimination de parasites de type helminthes. Quant aux lymphocytes Th17, ils favorisent la destruction de bactéries extracellulaires et de champignons.

reconnaissent les allo-antigènes. Deux modes d'action furent proposés : d'une part, un mécanisme passif de délétion des clones de lymphocytes T au niveau central [4], en phase avec la proposition de Burnet décrivant la sélection thymique comme mécanisme de tolérance naturelle vis-à-vis du soi [5] ; et, en périphérie, un mode d'action actif impliquant des cellules suppressives relativement mal identifiées [6]. Dans les deux cas, l'immunité néonatale était maintenue sous silence.

La tolérance centrale serait rendue possible par la perméabilité du thymus du nouveau-né aux précurseurs de cellules dendritiques du donneur contenus dans l'inoculum de cellules semi-allogéniques. Ces cellules dendritiques induiraient l'apoptose des clones de lymphocytes T allospécifiques émergeant au sein de cet organe. II s'est avéré ensuite que l'importance de ce mécanisme de délétion clonale induit chez le nouveau-né s'appliquait plus particulièrement aux lymphocytes $T C D 8^{+}$cytotoxiques du receveur présentant des disparités déterminées avec les molécules du complexe majeur d'histocompatibilité (CMH) de classe I du donneur [7].

En ce qui concerne les lymphocytes $\mathrm{TCD}^{+}$auxiliaires restreints pour la reconnaissance des molécules $\mathrm{CMH}$ de classe II, une déviation vers un phénotype Th2, plutôt qu'une absence de réponse, expliquerait l'incompétence des nouveau-nés à rejeter les cellules allogéniques [8]. II est connu que les réponses de type Thl, impliquées dans les défenses contre des infections bacté- riennes intracellulaires, sont caractérisées par une importante production d'interféron $\gamma$, une cytokine intervenant dans l'immunité à médiation cellulaire de type cytotoxique; au contraire, l'activité cytotoxique des réponses de type Th2 impliquées dans les défenses antiparasitaires, mais également dans les réactions allergiques, et caractérisées par une production d'interleukines-4 (IL-4), $-5,-10$ et -13 , est moindre (Figure 2). II fut ainsi décrit que les organes lymphoïdes du nouveau-né, contrairement à ceux de l'adulte, contiennent un nombre élevé de lymphocytes T CD4 ${ }^{+}$capables de produire de I'IL-4 en réponse à leur stimulation. L'IL-4 est une cytokine clé dans la polarisation des réponses immunes de type Th2. Ainsi, les lymphocytes T $\mathrm{CD} 4^{+}$matures du nouveau-né receveur se différenciant en cellules Th2 productrices d'IL-4 induiraient préférentiellement la différenciation des nouveaux lymphocytes $T$ anti-donneur en lymphocytes Th2 incapables de contribuer à l'élaboration d'une réponse cytotoxique allospécifique. L'équipe du $\operatorname{Pr}$ Lehman poursuivit cette remise en question du paradigme de la tolérance néonatale en démontrant que les souriceaux âgés de $24 \mathrm{~h}$ étaient capables d'activer 


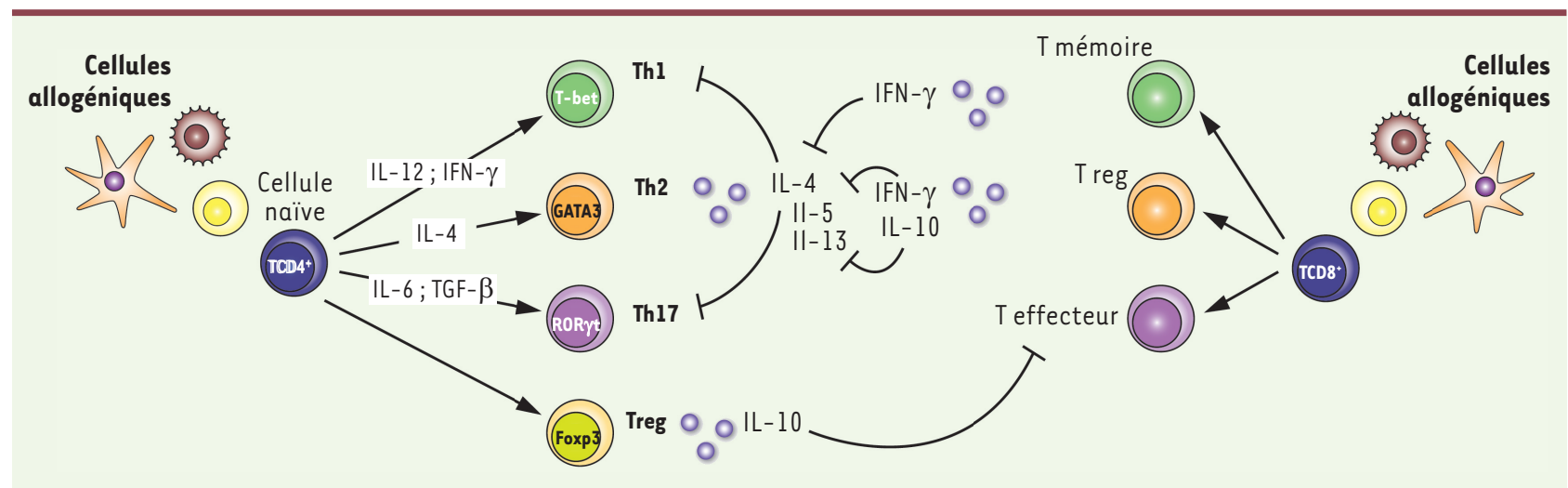

Figure 3. La régulation de la polarisation Th2 en réponse aux allo-antigènes au début de la vie. Le chimérisme créé par la persistance des cellules allogéniques implantées à la naissance conduit à une réponse dominante de type Th2 des lymphocytes T CD4 sécrétant rapidement des taux élevés d'IL-4. Cette cytokine permet un contrôle négatif du développement des réponses de type Thl et Th17. Des lymphocytes Treg peuvent également se développer par défaut et participer au contrôle négatif des lymphocytes T CD $8^{+}$effecteurs. En revanche, des lymphocytes T CD $8^{+}$de type mémoire produisant de I'IFN- $\gamma$, ou de type régulateur produisant de I'IFN- $\gamma$ et de l'IL-10, apparaissent et influencent à leur tour la polarisation Th2.

une réponse immune T auxiliaire vigoureuse et mixte [9]. L'induction d'une immunité de type Th2 se localisant préférentiellement dans la rate était relativement compatible avec un processus de tolérance active qui remplaça le modèle de suppression.

Chez la souris, la capacité des lymphocytes T CD $4^{+}$du nouveau-né à produire rapidement de forts taux d'IL-4, mais également d'IL13 , est imputable à un état particulier de méthylation de l'ADN conditionnant l'expression des gènes codant pour les cytokines Th2. Ainsi, dans les lymphocytes T CD4 ${ }^{+}$naïfs du nouveau-né, tant périphériques que thymiques, la région régulatrice non codante CNS-1, une séquence enhancer située au sein du locus Th2, serait particulièrement hypométhylée [10]. Cette caractéristique épigénétique intrinsèque aux lymphocytes $\mathrm{T} C D 4^{+}$du nouveau-né permit d'apprécier les conséquences d'une telle polarisation immunitaire. En effet, l'intensité de cette réponse de type Th2 peut être à l'origine d'un syndrome immunologique pathologique comportant une surproduction d'lg $\varepsilon$, une hyperplasie des organes lymphoïdes, une hyperéosinophilie et une augmentation de la production de cytokines de type Th2, telles que I'IL-4, I'IL-5, I'IL13 et I'IL-10, comme constaté lors de l'administration de cellules spléniques semi-allogéniques (Figure 1) [11]. L'IL-4 joue un rôle majeur dans l'induction de la tolérance néonatale. En effet, le traitement de nouveau-nés soumis à une immunisation semiallogénique par des anticorps neutralisant l'IL-4 interfère avec l'induction de tolérance, ce qui se traduit par une augmentation de l'activité T cytotoxique, une diminution du chimérisme cellulaire, une inhibition de la production d'lgદ et un rejet accéléré d'une greffe de même allogénicité [12].

\section{L’immunité néonatale}

La faiblesse des réponses de type Thl en début de vie

Les observations révolutionnaires de Lehman ont mis en avant le concept d'immunité néonatale, nuançant celui de la tolérance néo- natale. En fonction de l'adjuvant utilisé lors de l'exposition antigénique chez la souris, une réponse de type Thl pouvait également être induite en début de vie [9]. Il fut établi que la tolérance n'était pas le reflet d'une propriété intrinsèque du système immunitaire précoce, mais que la réponse dépendait du type de cellules présentatrices qui sensibilisait le système immunitaire à la rencontre antigénique. En effet, plusieurs protocoles vaccinaux, à l'aide de cellules dendritiques compétentes ou de leurs cytokines clés, comme l'IL-12p70 impliquée dans la polarisation de type Thl (Figure 2) [13], d'anticorps anti-CD40 provoquant la maturation des cellules dendritiques [14], de nucléotides CpG, de vaccins à $A D N$ ou de virus, renforcèrent l'idée que la réponse immunitaire néonatale primaire pouvait comporter tant des composantes de type Th1 que Th2 [15]. De nouvelles stratégies vaccinales visant les cellules dendritiques pouvaient ainsi être envisagées [16].

L'équipe du Pr Zaghouani précisa à l'échelle moléculaire comment la balance Th1/Th2 des réponses primaires induites chez les nouveau-nés de souris penche finalement en faveur d'une réponse secondaire de type Th2 [17]. II décrivit que, jusqu'au $8^{e}$ jour de vie chez la souris, l'absence, lors d'une stimulation antigénique primaire, de cellules dendritiques de type CD1l $c^{+} C D 8 \alpha^{+} C D 4^{-}$productrices d'IL-12p70, permettait l'expression de la chaîne $\alpha$ l du récepteur à l'IL-13 (IL-13R $\alpha$ I), qui s'hétérodimérise avec la chaîne $\alpha$ du récepteur à I'IL-4 à la surface des lymphocytes de type Th1. Lors d'une stimulation antigénique secondaire, I'IL-4 produite par les lymphocytes Th2 induit l'apoptose des cellules Thl via ce récepteur hétérodimérique IL-4R $\alpha / I L-13 R \alpha 1$, ce qui explique le biais Th2 de l'immunisation néonatale [18]. Ainsi, un défaut 
(confirmé chez l'homme [16]) de cellules dendritiques capables de fournir de manière optimale l'IL-12p70, comme la sécrétion rapide par les cellules Th2 d'IL-4 capable d'induire l'apoptose des cellules Thl, peuvent expliquer la faiblesse des réponses de type Thl en début de vie (Figure 3).

\section{Un défaut de réponse Th17}

Un défaut fonctionnel a également été décrit chez les nouveaunés de souris [19] en ce qui concerne les réponses de type Th17 impliquées dans la défense contre des agents pathogènes de types fongiques ou des bactéries extracellulaires, et qui jouent également un rôle pathologique dans des réponses auto-immunes comme l'encéphalomyélite expérimentale ( $દ A \varepsilon)$. Un mécanisme de régulation de la balance Th2/Th17 a été proposé. En effet, il fut démontré que I'IL-4 produite chez des nouveau-nés immunisés contre des cellules spléniques semi-allogéniques exerçait une régulation négative sur le développement d'une réponse de type Th17 spécifique des allo-antigènes et impliquée dans la cytotoxicité des cellules chimériques du donneur [20]. Un effet similaire d'induction d'apoptose des lymphocytes Th17 via leurs récepteurs à I'IL-4 ou à I'IL-13 [21] - autre cytokine fortement exprimée par les lymphocytes T CD $4^{+}$du nouveau-né -, doit encore être démontré (Figure 3).

\section{La tolérance néonatale :} signature de cellules régulatrices?

La tolérance immunitaire est un processus qui peut se maintenir tout au long de la vie, tant au niveau central qu'au niveau périphérique. Le statut immunitaire du nouveau-né est particulièrement enclin au développement de cellules régulatrices (Treg) capables de limiter toutes les cellules qui gèrent l'inflammation : les cellules T CD $8^{+}$, les cellules T CD4 ${ }^{+}$de type Th1, de type Th17 et de type Th2 (réponses Th2 excessives pouvant être à l'origine de pathologies allergiques).

Tout d'abord, Streilein démontra que des lymphocytes T suppresseurs transférables étaient impliqués dans le processus de tolérance néonatale chez des animaux présentant des disparités au niveau des molécules CMH de classe II [22], et ce par un processus dépendant de I'IL-4 et de I'IL-10. Ensuite, l'équipe de Gao montra que des lymphocytes $T$ régulateurs $C D 4^{+} C D 25^{+}$étaient capables d'atténuer l'alloréactivité des lymphocytes $\mathrm{T} \mathrm{CD8}^{+}$en diminuant leur activité cytotoxique [23]. Enfin, il fut établi que des thymocytes ou des splénocytes CD $4^{+}$CD $8^{-}$Foxp $3^{-}$de nouveau-nés de souris, et non d'adultes, se différenciaient en cellules Treg $C D 4^{+} F o x p 3^{+}$en réponse à une simple stimulation de leur TCR ( $T$ cell receptor) et du récepteur CD28, sans qu'il soit besoin d'une source extrinsèque de cytokines polarisantes comme I'IL-2 ou le TGF- $\beta$ (transforming growth factor) (Figure 2). Ainsi, durant la première semaine de vie, les lymphocytes $T$ sont intrinsèquement enclins à se différencier en lymphocytes Treg exprimant de manière stable le facteur de transcription Foxp3 (forkhead box P3) et exerçant des fonctions suppressives [24].
Chez la souris, des cellules B néonatales, plus particulièrement les lymphocytes $B-1 C D 19^{+} C D 5^{+}$, produisent d'importantes quantités d'IL-10 comparativement aux lymphocytes $B$ adultes en réponse à la stimulation des récepteurs Toll-like (TLR) -2, -4 et -9 . Elles peuvent ainsi empêcher la production d'IL-12p70 et l'augmentation de l'expression des molécules de costimulation exprimées par les cellules dendritiques néonatales [25]. De plus, en réponse au LPS (lipopolysaccharide), ces lymphocytes $B$ régulateurs de nouveau-né peuvent exercer des fonctions suppressives sur une réponse adaptative de type Thl dirigée contre des allo-antigènes. En revanche, in vivo, ces Breg néonataux producteurs d'IL-10 ne seraient pas impliqués directement dans l'induction de tolérance de transplantation [26].

Les lymphocytes T CD8 ${ }^{+}$ont la capacité de contrôler l'amplitude des réponses de type Th2 dirigées contre des pathogènes ou des allo-antigènes. Les mécanismes pouvant rendre compte de ce phénomène peuvent impliquer leur production d'IFN- $\gamma$ ou d'IL-10, une cytotoxicité envers les lymphocytes T CD4 $4^{+}$et les cellules dendritiques, une altération de l'expression de leurs molécules costimulatrices ou, également, l'induction d'une production d'IL-12p70 et d'IL-18, cytokines polarisant la réponse de type Thl [27]. De plus, des lymphocytes $T$ CD8 ${ }^{+}$sensibilisés aux alloantigènes à la naissance sont dépourvus d'activité cytotoxique dirigée contre les cellules allogéniques du donneur, mais possèdent la capacité de contrôler la plupart des paramètres de la réponse biaisée de type Th2, comme la sécrétion de cytokines de type Th2 (IL-4 et IL-5), la production d'IgE et l'hyperéosinophilie des organes lymphoïdes [28]. Cette régulation de la pathologie de type Th2 s'exerce, notamment, par le biais de lymphocytes $\operatorname{CD} 8^{+} C D 25^{+}$Treg exprimant le facteur de transcription Foxp3 et sécrétant de I'IFN- $\gamma$ et de I'IL-10 (Figure 3) [29].

Un autre mécanisme de contrôle des pathologies de type Th2 par les lymphocytes T CD $8^{+}$peut également s'établir indépendamment d'une reconnaissance restreinte de leur TCR [30]. Des lymphocytes T CD8 ${ }^{+}$ mémoires ou Treg ont la capacité d'empêcher le développement de pathologies inflammatoires de type Th2 induites par des antigènes contre lesquels ils ne sont pas spécifiques [31]. Ainsi, il fut démontré que des lymphocytes $\mathrm{T} C D 8^{+}$activés à la naissance par des allo-antigènes de manière à sécréter de forts taux d'IFN- $\gamma$, ont le pouvoir de contrôler le développement d'un asthme déclenché ultérieurement lors d'une exposition à l'ovalbumine de poulet [32]. Ce phénomène est particulièrement important dans le 
cadre du contrôle de l'incidence accrue des maladies allergiques observées en début de vie et pourrait expliquer la corrélation inverse qui existe entre l'exposition précoce à des pathogènes et le développement ultérieur d'allergies.

\section{La rupture de la tolérance néonatale et ses conséquences}

Tant chez l'homme que chez la souris, il existe une forme naturelle d'exposition aux allo-antigènes qui peut se manifester durant la vie fœtale et se poursuivre pendant l'allaitement. Des cellules d'origine maternelle exprimant des allo-antigènes non hérités NIMA (nonherited maternal alloantigens) peuvent être transportées à travers le placenta ou dans le lait maternel, et établir ainsi chez le nouveau-né un microchimérisme maternel qui influencera l'immunité adaptative de ce nouveau-né [33]. En effet, en fonction de la nature et de la dose des antigènes NIMA, s'établira soit une induction de tolérance via le développement de Treg pour les fortes doses [34] soit, au contraire, pour les faibles doses de NIMA, l'activation de réponses cytotoxiques de type Thl pouvant mener au développement d'un diabète auto-immun [35]. Nous posons donc l'hypothèse selon laquelle l'établissement d'un microchimérisme maternel favorisé par le statut particulier de la tolérance néonatale [36] est associé à une réponse adaptative de type Th2, de faibles réponses Thl et Th17 [37] et la présence de lymphocytes $\mathrm{T} C D 8^{+}$faiblement cytotoxiques. Cependant, le microenvironnement peut provoquer une inhibition de cette réponse polarisée de type Th2, en favorisant l'émergence de lymphocytes T CD8 ${ }^{+}$mémoires ou régulateurs, capables de contrôler le développement de pathologies allergiques, mais dont la conséquence serait le développement d'une réponse Th17 participant à l'élimination du microchimérisme maternel et pouvant être à l'origine de pathologies auto-immunes (Figure 3). $\diamond$

\section{SUMMARY}

Neonatal tolerance to alloantigens

In early life, our immune system has the characteristics of tolerance to alloantigens. During this period, our adaptive immunity is not at rest. On the contrary, it is polarized so as to promote the activation of Th2-type T cell response at the expense of cytotoxic Th1-or Th17-type responses. This may explain the vulnerability of infants to aggression by pathogens, their increased sensitivity to develop allergic diseases or their poor responses to some vaccines. Exposure to environmental factors will modify that neonatal tolerance due to its control by other actors and will thus have potential repercussions on the subsequent onset of allergies or autoimmune diseases. $\diamond$

\section{LIENS D’INTÉRÊT}

L'auteur déclare n'avoir aucun lien d'intérêt concernant les données publiées dans cet article.

\section{REMERCIEMENTS}

Michel Goldman, Daniel Abramowicz, Vincent Donckier, Alain Le Moine, Brigitte Adams, Aurore Dubois et Isabelle Debock ont contribué aux travaux du labora- toire auxquels il est fait référence. Les travaux du laboratoire ont bénéficié du soutien du Fonds national de la recherche scientifique (FNRS Belgique), du projet NEOVAC de la région wallonne en partenariat avec GSK-bio et d'un pôle d'attraction interuniversitaire de la police scientifique fédérale belge. Illustrations, Luc Segers.

\section{RÉFÉRENCES}

1. Owen RD. Immunological consequences of vascular anastomoses between bovine twins. Science $1945 ; 102: 400$.

2. Cannon JA, Longmire WP. Studies of successful skin homografts in the chicken. Ann Surg 1952; $135: 60-8$.

3. Billingham RE, Brent L, Medawar PB. Actively acquired tolerance of foreign cells. Nature $1952 ; 172: 603$.

4. Gammon G, Dunn K, Shastri N, et al. Neonatal T-cell tolerance to minimal immunogenic peptides is caused by clonal inactivation. Nature $1986 ; 319$ : 413-5.

5. Burnet FM. Immunological recognition of self. Science 1961 ; 133 : 307-11.

6. Dorsch S, Roser B. T cells mediate transplantation tolerance. Nature 1975 ; $258: 233-5$.

7. Feng HM, Glasebrook AL, Engers HD, et al. Clonal analysis of T cell unresponsiveness to alloantigens induced by neonatal injection of $\mathrm{Fl}$ spleen cells into parental mice. J Immunol $1983 ; 131: 2165-9$.

8. Powel TJ, Streilein JW. In vitro suppression of cytotoxic T cell generation by lymphocytes from mice rendered neonatally tolerant of class II MHC alloantigens. Transplantation $1991 ; 52$ : 383-6.

9. Forsthuber T, Yip HC, Lehman PV. Induction of Thl and Th2 immunity in neonatal mice. Science $1996 ; 271: 1728-30$.

10. Rose S, Lichtenheld M, Foote MR, et al. Murine neonatal CD4 ${ }^{+}$cells are poised for rapid Th2 effector-like function. J Immunol 2007 ; 178 : 2667-78.

11. Le Moine A, Flamand V, de Lavareille A, et al. Hypereosinophilic syndrome induced by neonatal immunization against MHC class II alloantigen: critical role of IL-4. Eur J Immunol $2002 ; 32$ : 174-81.

12. Donckier V, Wissing M, Bruyns $C$, et al. Critical role of interleukin 4 in the induction of neonatal transplantation tolerance. Transplantation $1995 ; 59$ : 1571-6.

13. Donckier V, Flamand V, Desalle F, et al. IL-12 prevents neonatal induction of transplantation tolerance in mice. Eur J Immunol 1998 ; 28 : 1426-30.

14. Flamand V, Donckier V, Demoor F, et al. CD40 ligation prevents neonatal induction of transplantation tolerance. J Immunol 1998 ; 160 : 4666-9.

15. Lotteau V. La période de tolérance néonatale existe-t-elle ? Med Sci (Paris) $1996 ; 12: 983-7$.

16. Goriely $S$, De Wit $D$, Flamand $V$, et al. Les réponses immunes à médiation cellulaire chez le nouveau-né : vers des nouvelles stratégies vaccinales ciblant les cellules dendritiques? Med Sci (Paris) 2001 ; 17 : 1337-41.

17. Adkins B, Du RQ. Newborn mice develop balanced Th1/Th2 primary effector responses in vivo but are biased to Th2 secondary responses. J Immunol $1998 ; 160: 4217-24$.

18. Lee H-H, Hoeman CM, Hardaway JC, et al. Delayed maturation of an IL-12producing dendritic cells subset explains the early Th2 bias in neonatal immunity. J Exp Med 2008 ; $205: 2269-80$.

19. Hofstetter HH, Kovalovsky A, Shive CL, et al. Neonatal induction of myelinspecific Thl/Th17 immunity does not result in experimental autoimmune encephalomyelitis and can protect against the disease in adulthood. J Neuroimmunol $2007 ; 187: 20-30$.

20. Debock I, Delbauve S, Dubois A, et al. Th17 alloimmunity prevents neonatal establishment of lymphoid chimerism in IL-4-deprived mice. Am J Transplant $2012 ; 12: 81-9$.

21. Newcomb D, Zhou W, Moore ML, et al. A functional IL-13 receptor is expressed on polarized murine CD4+ Th17 cells and IL-13 signaling attenuates Th17 cytokine production. J Immunol 2009 ; 182 : 5317-21.

22. Powell TJ, Streilein JW. In vitro suppression of cytotoxic T cell generation by lymphocytes from mice rendered neonatally tolerant of class II MHC alloantigens. Transplantation $1991 ; 52$ : 383-6.

23. Gao $Q$, Rouse $T M$, Kazmerzak $K$, et al. $C D 4^{+} C D 25^{+}$cells regulate $C D 8$ cell anergy in neonatal tolerant mice. Transplantation 1999; $68: 1891-7$.

24. Wang G, Miyahara Y, Guo Z, et al. «Default » generation of neonatal regulatory T cells. J Immunol $2010 ; 185$ : 71-8. 


\section{RÉFÉRENCES}

25. Sun CM, Deriaud $\varepsilon$, Leclerc C, et al. Upon TLR9 signaling, $C D 5^{+} B$ cells control the IL-12-dependent Th1-priming capacity of neonatal DCs. Immunity $2005 ; 22: 467-77$

26. Walker WE, Goldstein $\mathrm{Dr} B$ cells are dispensable for neonatal transplant tolerance induction. Transplantation $2009 ; 88: 874-8$.

27. Vukmanovic-Stejic M, Thomas MJ, Noble A, et al. Specificity, restriction and effector mechanisms of immunoregulatory CD8 T cells. Immunology $2001 ; 102: 115-22$.

28. Adams B, Nagy N, Paulart F, et al. CD8 ${ }^{+}$T lymphocytes regulating Th2 pathology escape neonatal tolerization. J Immunol $2003 ; 171: 5071-6$.

29. Adams B, Dubois A, Delbauve $S$, et al. Expansion of regulatory $C D 8^{+} C D 25^{+} T$ cells after neonatal alloimmunization. Clin Exp Immunol 2010 ; 163 : 354-61.

30. De Lavareille A, Prigogine $C$, Paulart F, et al. Regulatory role of host $C D 8^{+} T$ lymphocytes in experimental graft-versus-host disease across a single major histocompatibility complex class II incompatibility. Transplantation $2005 ; 80: 1293-9$.

31. Berg RE, Crossley $\varepsilon$, Murray $S$, et al. Memory CD8 ${ }^{+} T$ cells provide innate immune protection against Listeria monocytogenes in the absence of cognate antigen. J Exp Med 2003 ; 198 : 1583-93.

32. Dubois A, Deruytter N, Adams B, et al. Regulation of Th2 responses and allergic inflammation through bystander activation of CD8+ $T$ lymphocytes in early life. J Immunol $2010 ; 185: 884-91$.
33. Andrassy J, Kusaka S, Jankowska-Gan $\varepsilon$, et al. Tolerance to noninherited maternal MHC antigens in mice. J Immunol 2003 ; 171 : 5554-61.

34. Mold JI, Michaëlsson J, Burt TD, et al. Maternal alloantigens promote the development of tolerogenic fetal regulatory T cells in utero. Science 2008 ; $322: 1562-5$

35. Roy $\varepsilon$, Leduc M, Guegan S, et al. Specific maternal microchimeric T cells targeting fetal antigens in beta cells predispose to auto-immune diabetes in the child. J Autoimmun $2011 ; 36$ : 253-62.

36. Dutta P, Molitor-Dart M, Bobadilla JL, et al. Microchimerism is strongly correlated with tolerance to noninherited maternal antigens in mice. Blood $2009 ; 114: 3578-87$.

37. Debock I, Flamand V. Th2 alloimmunity counteracts Th17-type response in the neonatal establishment of lymphoid chimerism. Chimerism $2011 ; 2$ : 117-19.

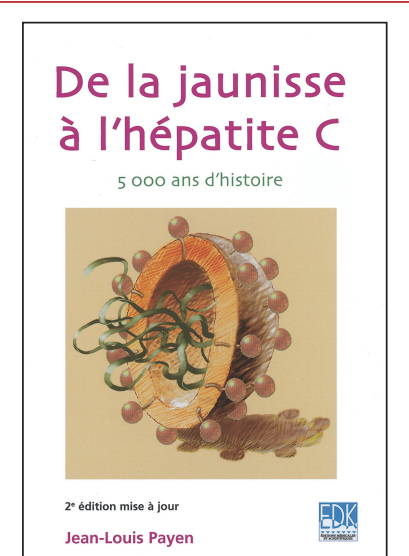

ISBN : 978-2-8425-4136-1 128 pages a jaunisse est un symptôme facilement identifiable ; il paraissait bien naturel que l'homme, confronté à une modification de la couleur de ses yeux et de sa peau ait de tous temps recherché les causes de cette transformation.

II n'est donc pas surprenant que le premier traité de médecine, écrit 3000 ans avant J.C. par un médecin sumérien, décrive déjà la jaunisse. À chaque époque de l’histoire de la médecine, les praticiens, influencés par les concepts médicaux de leur temps, attribuèrent une ou plusieurs explications particulières à ce symptôme. Ainsi, du démon Ahhâzu des Sumériens à la sophistication des biotechnologies qui permirent la découverte du virus de l'hépatite C, le lecteur cheminera sur une période de 5000 ans au travers des différents continents.

Ici encore, l'histoire se révèle une formidable source de réflexion : le foie souvent impliqué dans l'apparition des jaunisses est-il le siège de l’âme?

Les expérimentations humaines chez des volontaires ou chez des enfants handicapés mentaux étaient-elles justifiées pour permettre la découverte des virus des hépatites?

Le formidable développement de la transfusion sanguine, des vaccinations, mais aussi de la toxicomanie explique-t-il les épidémies d'hépatites du XXe siècle?

Autant de questions qui sont abordées dans ce livre passionnant et accessible à tous.

UU À retourner à EDK, 25, rue Daviel - 75013 Paris - Tél. : 0158101905 - Fax : 0143293262 - E-mail : edk@edk.fr

NOM :

Prénom :

Adresse :

Code postal :

Ville :

Pays :

Fonction :

Je souhaite recevoir l'ouvrage De la jaunisse à l'hépatite $\mathbf{C , ~} \mathbf{5} \mathbf{0 0 0}$ ans d'histoire : $12 €+3 €$ de port $=\mathbf{1 5} € \mathbf{T T C}$

en ................ exemplaire, soit un total de $€$

U Par chèque, à l'ordre de $\mathbf{E} \mathbf{D} \mathbf{K}$

$\square$ Par carte bancaire : $\square$ Visa $\square$ Eurocard/Mastercard

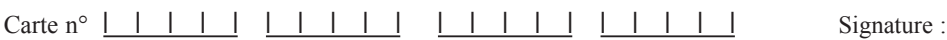

Date d'expiration :

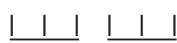

$\mathrm{N}^{\circ}$ de contrôle au dos de la carte :

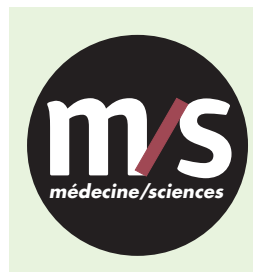

Tarifs d'abonnement $m / s-2014$

$>$ Grâce à $m / s$, vivez en direct les progrès des sciences biologiques et médicales

Abonnez-vous

à médecine/sciences
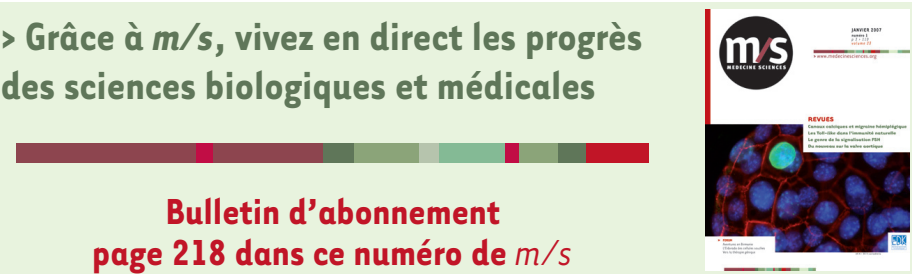